\title{
Mass-loading, pile-up, and mirror-mode waves at comet 67P/Churyumov-Gerasimenko
}

\author{
M. Volwerk ${ }^{1}$, I. Richter ${ }^{2}$, B. Tsurutani ${ }^{3}$, C. Götz ${ }^{2}$, K. Altwegg ${ }^{4}$, T. Broiles ${ }^{5}$, J. Burch ${ }^{5}$, C. Carr ${ }^{6}$, E. Cupido ${ }^{6}$, \\ M. Delva ${ }^{1}$, M. Dósa ${ }^{7}$, N. J. T. Edberg ${ }^{8}$, A. Eriksson ${ }^{8}$, P. Henri ${ }^{7}$, C. Koenders ${ }^{2}$, J.-P. Lebreton ${ }^{9}$, K. E. Mandt ${ }^{5}$, \\ H. Nilsson ${ }^{10}$, A. Opitz ${ }^{7}$, M. Rubin ${ }^{4}$, K. Schwingenschuh ${ }^{1}$, G. Stenberg Wieser ${ }^{10}$, K. Szegö ${ }^{7}$, C. Vallat ${ }^{11}$, X. Vallieres ${ }^{9}$, \\ and K.-H. Glassmeier ${ }^{2}$ \\ ${ }^{1}$ Space Research Institute, Austrian Academy of Sciences, Graz, Austria \\ ${ }^{2}$ Institute for Geophysics and Extraterrestrial Physics, TU Braunschweig, Germany \\ ${ }^{3}$ California Institute of Technology, Pasadena, California, USA \\ ${ }^{4}$ Physikalisches Institut, University of Bern, Bern, Switzerland \\ ${ }^{5}$ Southwest Research Institute, San Antonio, Texas, USA \\ ${ }^{6}$ Space and Atmospheric Physics Group, Imperial College London, London, UK \\ ${ }^{7}$ Wigner Research Centre for Physics, Institute for Particle and Nuclear Physics, Hungarian Academy \\ of Sciences, Budapest, Hungary \\ ${ }^{8}$ Swedish Institute of Space Physics, Uppsala, Sweden \\ ${ }^{9}$ Laboratoire de Physique et Chimie de l'Environnement et de l'Espace, Orleans, France \\ ${ }^{10}$ Swedish Institute of Space Physics, Kiruna, Sweden \\ ${ }^{11}$ Rosetta Science Ground Segment, European Space Astronomy Centre, Madrid, Spain \\ Correspondence to: M. Volwerk (martin.volwerk@oeaw.ac.at)
}

Received: 16 October 2015 - Revised: 1 December 2015 - Accepted: 6 December 2015 - Published: 15 January 2016

\begin{abstract}
The data from all Rosetta plasma consortium instruments and from the ROSINA COPS instrument are used to study the interaction of the solar wind with the outgassing cometary nucleus of 67P/Churyumov-Gerasimenko. During 6 and 7 June 2015, the interaction was first dominated by an increase in the solar wind dynamic pressure, caused by a higher solar wind ion density. This pressure compressed the draped magnetic field around the comet, and the increase in solar wind electrons enhanced the ionization of the outflow gas through collisional ionization. The new ions are picked up by the solar wind magnetic field, and create a ring/ringbeam distribution, which, in a high- $\beta$ plasma, is unstable for mirror mode wave generation. Two different kinds of mirror modes are observed: one of small size generated by locally ionized water and one of large size generated by ionization and pick-up farther away from the comet.
\end{abstract}

Keywords. Space plasma physics (charged particle motion and acceleration; nonlinear phenomena; waves and instabilities)

\section{Introduction}

The theory of the interaction of an outgassing comet with the solar wind magnetoplasma started with the explanation of the formation and physics of the cometary ion tails by Biermann (1953) and Alfvén (1957). With the beginning of the space age and spacecraft-flybys of comets in the last century, e.g. VEGA 1, 2, Giotto, ICE, Sakigake and Suisei by comet 1P/Halley, Giotto at 26P/Grigg-Skjellerup and ICE at 21P/Giacobini-Zinner, much has been learned about the various physical processes taking place in the plasma around the outgassing cometary nucleus.

In the current century, on 20 January 2014 the Rosetta spacecraft (Glassmeier et al., 2007) was woken up after 18 months of hibernation, and the spacecraft cruised towards its rendezvous with comet 67P/Churyumov-Gerasimenko (67P/CG). On 6 August 2014 Rosetta arrived at its target, and started its escort phase, following the comet along its orbit from pre- to past-perihelion. 67P/CG's perihelion was on 13 August 2015. 
In this paper the data from the Rosetta Plasma Consortium instruments (RPC, Carr et al., 2007) are used to study the interaction of the outgassing nucleus of comet $67 \mathrm{P} / \mathrm{CG}$ and the solar wind magnetoplasma at a time when the comet is closing in on its perihelion. Unlike the previous missions mentioned above, Rosetta does not perform a quick flyby of the comet, but remains at the comet, moving at a very slow pace of $\sim 1 \mathrm{~m} \mathrm{~s}^{-1}$. This means that Rosetta RPC can follow the development of the interaction of the solar wind with the increasingly more actively outgassing nucleus as comet 67P/CG heads towards perihelion, and the decreasing activity after perihelion.

After initial arrival a new phenomenon was found, now called the "singing comet" (Richter et al., 2015); 40 mHz waves generated by a cross-field current instability created by freshly ionized, not yet magnetized water ions within the Larmor sphere (sphere with radius of 1 Larmor radius, Sauer et al., 1998) of the comet. At that time, these newly created ions also indicated the "birth of a magnetosphere" (Nilsson et al., 2015a) for which the spatial distribution of the lowenergy plasma was discussed by Edberg et al. (2015b). However, "conventional signatures" such as Alfvén waves or cyclotron waves were not observed.

Later in the mission, with comet 67P/CG approaching its perihelion, the activity of the nucleus increased significantly. Various strong outbursts were observed by the Rosetta NAVCAM, see Fig. 1, which mainly shows reflected sunlight on dust grains, and these might significantly influence the plasma interactions. Rotundi et al. (2015) discussed the link between gas and dust emissions. Indeed, in the second half of July 2015, the outgassing of the nucleus was so strong that a diamagnetic cavity was created which extended well past the $\sim 180 \mathrm{~km}$ distance of Rosetta from comet 67P/CG (Glassmeier et al., 2015; Götz et al., 2015, see also http://blogs.esa.int/rosetta/2015/08/11/ comets-firework-display-ahead-of-perihelion/). Koenders et al. $(2013,2014)$ have predicted distances of $\sim 25 \mathrm{~km}$ for the diamagnetic cavity distance under quiet conditions. Such strong outburst conditions have not been modelled yet. In a diamagnetic cavity the outflowing neutral gas and plasma is strong enough to keep the solar wind and its embedded magnetic field at bay, pushing it away from the nucleus (see e.g. Cravens and Gombosi, 2004). This creates a magnetic field-free region around the comet. However, the Rosetta RPC magnetometer did still measure a very small magnetic field, which is an indication for the not-fully corrected offsets of the magnetometer, which can be either inherent or arise from stray fields from the spacecraft. In this paper the measured fields have been used to correct the offset.

In this paper a first overview and discussion is given of the events taking place on 6 and 7 June 2015. There is a $\sim 6 \mathrm{~h}$ quasi-periodic variation in the neutral and plasma density (Hässig et al., 2015; Edberg et al., 2015b). First the effect of the mass loading on the induced magnetosphere is discussed, including magnetic field pile-up and draping, relating

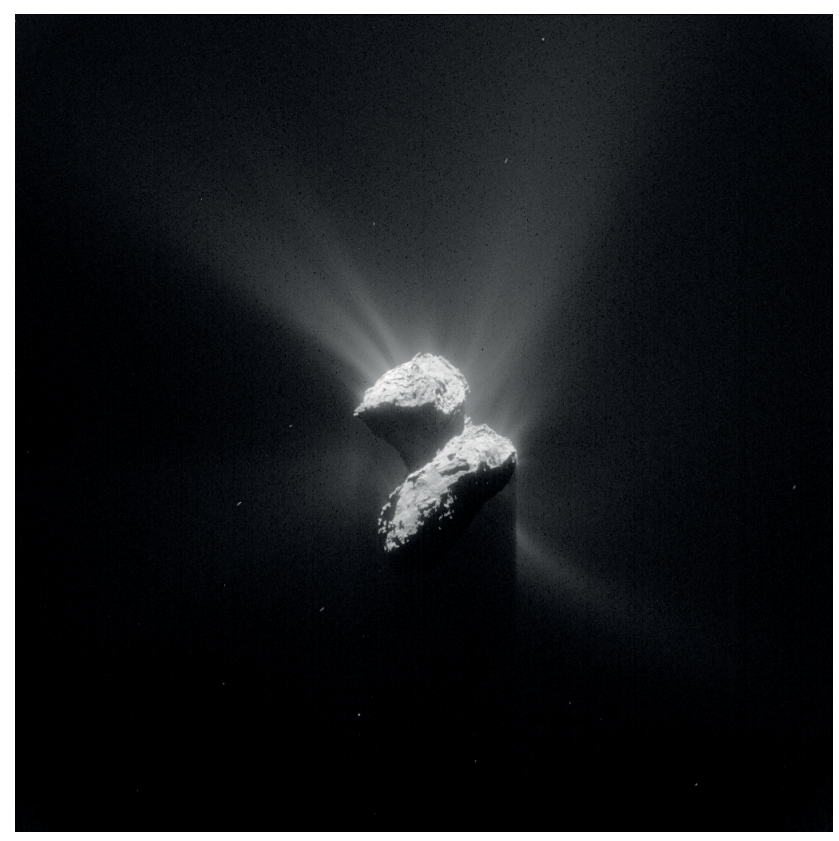

Figure 1. NAVCAM image of comet 67P/CG on 5 June 2015, showing the structuredness of the escaping dust from the nucleus. Credits: ESA/Rosetta/NAVCAM - CC BY-SA IGO 3.0.

it to variations in the solar wind. Second, the behaviour of the freshly created ions and the resulting mirror-mode wave activity is investigated.

\section{Mass loading of the induced magnetosphere}

On 6 June 2015 there was a higher-than-usual gas-outflow from the comet, which loaded the induced magnetosphere with neutral gas and plasma. The combined data of the six instruments discussed below, for the 2-day interval of 6-7 June 2015 are shown in Fig. 2. From top to bottom the following is shown: the Ion and Electron Spectrometer (IES, Burch et al., 2006) time-energy spectrogram, the Ion Composition Analyser (ICA, Nilsson et al., 2006) time-energy spectrogram, the low-pass filtered magnetic field components in Cometocentric Solar EQuatorial $\left(\mathrm{CSEQ}^{1}\right)$ coordinates from the Magnetometer (MAG, Glassmeier et al., 2007); the magnetic field strength, the Mutual Impedance Probe (MIP, Trotignon et al., 2006) deduced electron densities; the LAngmuir Probe (LAP, Eriksson et al., 2006) P1 current, the IES ion and electron density; the Rosetta Orbiter Spectrometer for Ion and Neutral Analysis (ROSINA, Balsiger et al., 2007) Cometary Pressure Sensor (COPS) neutral density; the location of the spacecraft with respect to the comet; the IES ion velocity in CSEQ and the angles of the ion velocity with the radial direction to the comet and with the magnetic field direction.

\footnotetext{
${ }^{1}$ CSEQ: A cometocentric coordinate system with the $x$ axis pointing towards the Sun, the $z$ axis is aligned with the rotational axis of the Sun, and the $y$ axis completes the triad.
} 


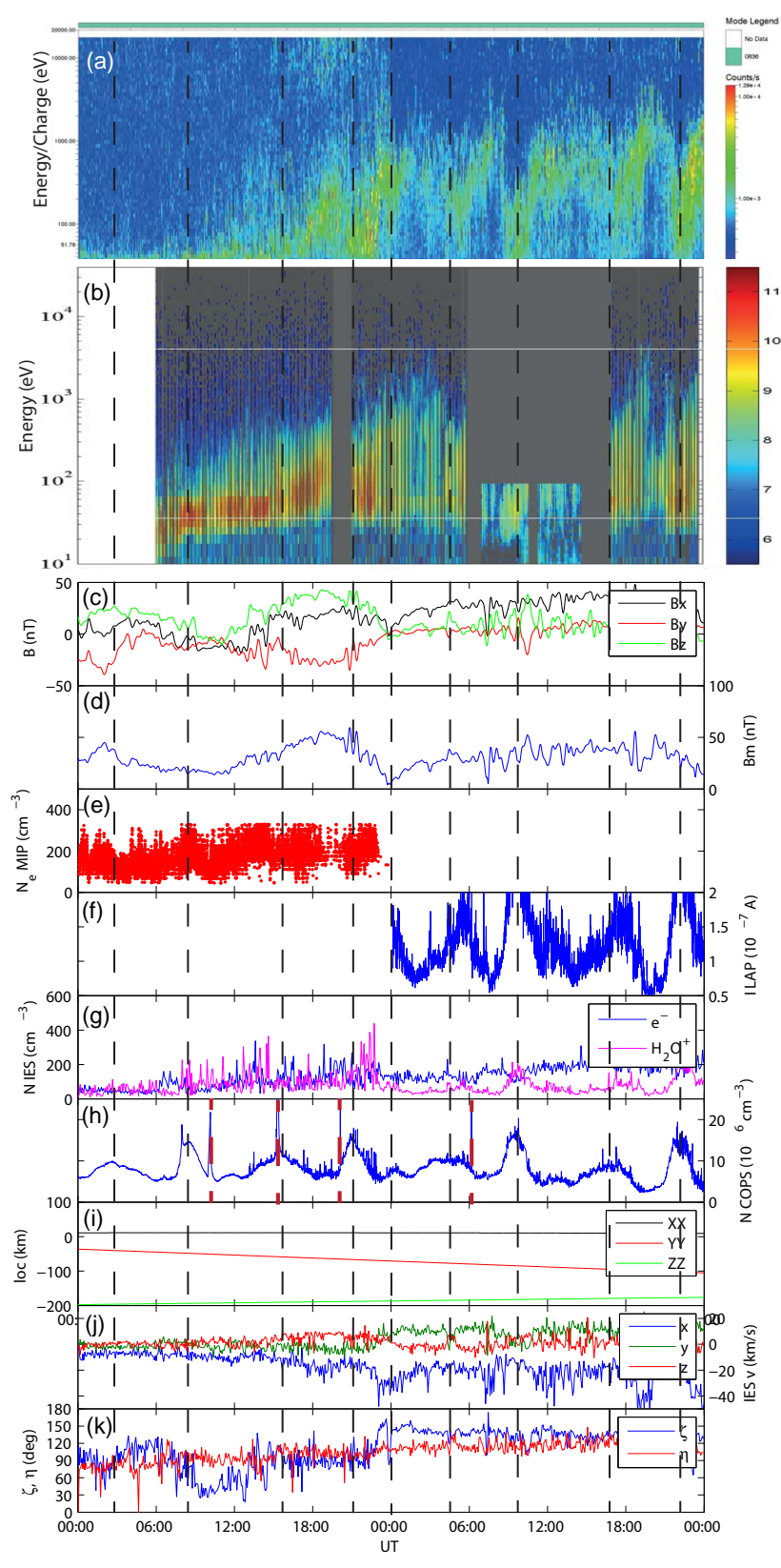

Figure 2. (a) The time-energy spectrogram of ion monitor of the IES instrument. (b) The time-energy spectrogram of the ICA instrument. The low cut-off during the interval on 7 June between 07:00 and 15:00 UT is caused by a mode change of the instrument. (c) The three components of the magnetic field in CSEQ coordinates. (d) The magnetic field strength. (e) The MIP-deduced electron density. (f) The LAP P1 current. (g) The deduced electron and ion density from the IES instrument. (h) The neutral density from ROSINA COPS. (i) The location of Rosetta in CSEQ coordinates. (j) The ion velocity components from the IES instrument. (k) The angle $\eta$ between the velocity vector and the radial direction to the comet and the angle $\zeta$ between the ion velocity vector and the magnetic field vector. The vertical lines are at 02:45, 08:30, 15:30, 21:00, 00:30, 04:15, 09:30, 16:45, and 22:00 UT.
In both the IES and the ICA, an increase in ion counts and energies in the ion channels starting at approximately 18:00 UT is seen. There is an increase in energy from $\sim 10 \mathrm{eV}$ to up to $\sim 500 \mathrm{eV}$ for both instruments, where IES seems to show a sawtooth-like behaviour with a quasi-period of around 4 to $6 \mathrm{~h}$ as shown in Fig. 2.

The neutral gas density measured by COPS of ROSINA is shown in Fig. 2h. A semi-periodic density fluctuation at a quasi-period of $\sim 6 \mathrm{~h}$, and a few maxima at $\sim 08: 30$ and $\sim$ 15:45 UT and a very strong peak at $\sim 21: 00$ UT are seen. The second and third bursts (vertical dashed lines) coincide well with the start of energy increases in the IES and ICA data in Fig. 2.

It is clear from comparing panels $\mathrm{a}, \mathrm{b}, \mathrm{d}$ and $\mathrm{g}$ in Fig. 2 that a severe change occurs in the environment around comet 67P/CG; the magnetic field strength starts to increase around 11:00 UT, when at the same time IES and ICA data show an increase in counts and energies of the ions.

As the total magnetic field strength increases, the fluctuations in the magnetic field are also enhanced: the field increases from average $\overline{\boldsymbol{B}} \approx 27 \mathrm{nT}$ with a standard deviation $\sigma \approx 11 \mathrm{nT}$ during 00:00 to $12: 00 \mathrm{UT}$ to $\overline{\boldsymbol{B}} \approx 41 \mathrm{nT}$ with $\sigma \approx 16 \mathrm{nT}$ during 12:00 to 24:00 UT. In the early hours of 7 June the magnetic field strength has returned to a lower value $\overline{\boldsymbol{B}} \approx 30 \mathrm{nT}$ with $\sigma \approx 12 \mathrm{nT}$, and the IES densities in Panel $\mathrm{e}$ return to the values as at the beginning of 6 June and the ion densities and the LAP P1 current follow the COPS neutral densities in Panel $\mathrm{f}$. It should be noted that near 24:00 UT on 6 June the magnetic field strength decreases to a very low value of $\boldsymbol{B}_{\mathrm{m}} \approx 4 \mathrm{nT}$.

There is an interesting correlation between the data from ROSINA COPS neutral density and the densities measured by the RPC instruments. In Fig. 2 the vertical dashed lines are coincident with the maxima in the COPS data, with the black dashed lines marking the "regular" $6 \mathrm{~h}$ maxima. The sharp density peaks at the maroon coloured dashed lines are artifacts created by reaction wheel offloading on the spacecraft. There appears to be a delay in the response in the IES timeenergy spectrogram to the increased neutral density. After a neutral density maximum, the count rate and the ion energy increase and drop just before a new neutral density maximum is reached again. This may be due to the ionization time, and will have consequences for when RPC-measurable ions can be observed after neutral injection. However, this is beyond the scope of this paper.

The solar wind transports magnetic fields from the Sun towards the comet. In the surroundings of the comet a conducting layer exists, created by ionization of the outflowing gas from the nucleus. As discussed by Alfvén (1957) the magnetic field cannot pass unimpeded through this region near the nucleus and gets hung-up, whereas the part of the field lines further away are still moving with solar wind velocity. This leads to two phenomena: near the nucleus the magnetic field will pile-up, i.e. increase in strength, as the field is delivered faster than it can be transported away. This creates 

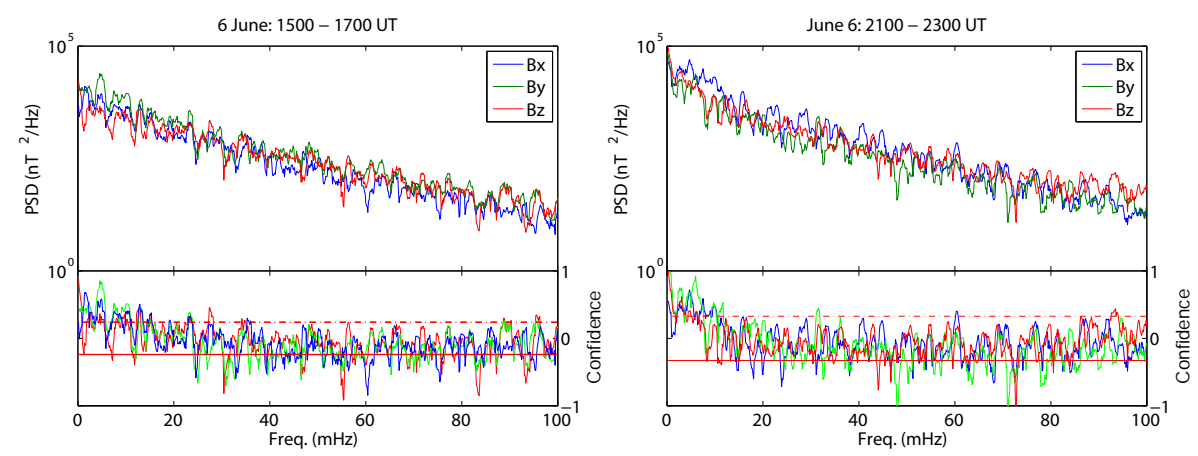

Figure 3. Left-top: power spectra of the three magnetic field components (in CSEQ coordinates) on 6 June between 15:00 and 17:00 UT. Left-bottom: fourth-order polynomial detrended spectra with $\pm 95 \%$ confidence levels indicated by horizontal solid and dash-dotted red lines. Top- and bottom-right: Same as left for 6 June 21:00-23:00 UT.

the so-called induced magnetosphere of the comet. Furthermore, the field lines wrap around the nucleus, get draped, because of the difference in velocity along the field line. These phenomena have been well studied during the flybys of other comets in the last century (see e.g. Smith et al., 1986; Riedler et al., 1986; McComas et al., 1987; Raeder et al., 1987; Israelevich et al., 1994; Israelevich and Ershkovich, 1994; Delva et al., 2014).

\subsection{Massloading at $\sim 15: 45 \mathrm{UT}$}

At $\sim 15: 45$ UT on 6 June, COPS shows a maximum in the neutral gas density in the quasi-periodic $\sim 6 \mathrm{~h}$ changes. IES shows an increase in energy and counts of the ions over the following 4 hours, however, note this signature looks different from what is happening after midnight on 7 June. The IES ion (electron) density, Fig. 2e, is rather peaked and strongly variable and reaches a maximum density at $\sim 14: 36$ ( 13:36) UT, which is most likely the result of the increased neutral density at $\sim 08: 30$ UT. After the $\sim 15: 45$ UT neutral density maximum the ion (electron) density starts to increase, with a slight maximum at $\sim 17: 40 \mathrm{UT}$.

With the increased plasma density a simultaneous increase in magnetic field strength $\boldsymbol{B}_{\mathrm{m}}$ is observed, see Fig. $2 \mathrm{~b}$. This could be a result of more magnetic pile-up because of the increased mass loading generating a layer with higher conductivity and thus a longer diffusion time. It is, however, unclear if an increase in ion density can actually lead to such a strong increase in magnetic field strength through increased hang-up. Volwerk et al. (2014) posited that a decrease in ion density at comet $1 \mathrm{P} /$ Halley could be the reason for the disappearance of the nested draped magnetic field between the flybys of Vega 1 and Vega 2. However, it is also quite possible that the increase in magnetic field strength and the increase in ion density are generated by an external source in the solar wind. This will be discussed in the next section.

Interestingly though, the situation is different from what was observed at comet 1P/Halley (see e.g. Gringauz et al., 1986; Neubauer et al., 1986), where the magnetic fluctua- tions disappeared in the pile-up region. At comet 67P/CG the magnetic fluctuations increase in the pile-up region.

With $\overline{\boldsymbol{B}} \approx 50 \mathrm{nT}$ the gyro frequency of water ions is $f_{c, \mathrm{H}_{2} \mathrm{O}} \approx 40 \mathrm{mHz}$. Spectral analysis of the interval 17:0019:00 UT on 6 June is performed and displayed in Fig. 3 top-left panel. The three components of the magnetic field are spectrally analysed (cf. McPherron et al., 1972) and displayed. In order to find the confidence level of the peaks, the spectra are fitted by a fourth-order polynomial, which is subtracted from the spectrum and from the residual (bottomleft panel) the $\pm 95 \%$ confidence level is determined (see e.g. Bendat and Piersol, 1966), shown as a red solid and dashdotted line. The spectrum shows that the strongest (highest PSD) component is $B_{y}$, there is a strong peak at $\sim 4.7 \mathrm{mHz}$ in $B_{x}$ and $B_{y}$ and a peak at $\sim 5.5 \mathrm{mHz}$ in $B_{z}$, and mutual second and third peaks at $\sim 7.7$ and $\sim 13 \mathrm{mHz}$. No significant signal is found at the water ion gyro frequency.

\subsection{Massloading at $\sim 21: 00 \mathrm{UT}$}

At $\sim 21: 00$ UT COPS showed another maximum in neutral gas density. The IES ion density increases with a maximum $N_{\mathrm{i}} \approx 430 \mathrm{~cm}^{-3}$ at $\sim 22: 40 \mathrm{UT}$, after which it quickly returns to pre-event values around $N_{\mathrm{i}} \approx 50 \mathrm{~cm}^{-3}$. Spectral analysis of the interval 21:00 to 23:00 UT of 6 June shows (see Fig. 3 right panels) that the strongest component is $B_{x}$; there is a first mutual peak at $\sim 2.8 \mathrm{mHz}$, a second, stronger, peak in $B_{x}$ is found at $\sim 4.7 \mathrm{mHz}$, whereas for $B_{y}$ a second peak is found at $\sim 6.0 \mathrm{mHz}$ and for $B_{z}$ a second peak is found at $\sim 5.4 \mathrm{mHz}$. There seems to be little common behaviour of the three magnetic field components.

\subsection{Ion motion}

The deduced ion velocities from the IES instrument are shown in Fig. 2h. On 6 June the ion $\left(\mathrm{H}_{2} \mathrm{O}^{+}\right)$velocity is around $\overline{\boldsymbol{v}} \approx(-12,-1,2) \mathrm{km} \mathrm{s}^{-1}$, with the magnitude of the components increasing when the mass loading starts around 16:00 UT (but the increase in magnetic field strength already 
starts about 2 hours earlier). Mainly $v_{x}$ and $v_{z}$ (in CSEQ coordinates) increase in magnitude with strongest change in $v_{x}$. After the increase in density and the increase in magnetic field strength disappear, just before midnight, $v_{z}$ returns to pre-mass-loading values, but $v_{x}$ and $v_{y}$ strongly increase in magnitude with $\overline{\boldsymbol{v}} \approx(-23,10,1) \mathrm{km} \mathrm{s}^{-1}$, lasting for many hours. This means that the ions are mainly moving antisunward as discussed by Nilsson et al. (2015b).

In order to determine the propagation direction of the ions the angle $\eta$ with the radial direction to the centre of the comet (red line) is calculated, as well as the angle $\zeta$ of the velocity with the local magnetic field (blue line) in Fig. 2i. Basically, over the whole of 6 June the ions are moving perpendicular to the radial direction to the comet and nearly perpendicular to the magnetic field, apart from 09:00-15:00 UT, which is related to the rotation of the magnetic field discussed further below.

Near midnight, after the enhanced mass-loading, the situation changes: the ions are accelerated in the $X-Y$ plane and move still mainly perpendicular to the radial vector with $\eta \approx 110^{\circ}$. However, the angle with respect to the magnetic field increases to $\zeta \approx 140^{\circ}$. The latter is what one would expect for newly formed ions being accelerated by the motional electric field (see also Broiles et al., 2015) whilst having an initial velocity at ionization, starting their gyration around the magnetic field, creating a ring-beam distribution, which can be unstable for mirror-mode waves (Hasegawa, 1969; Tsurutani et al., 1982; Gary, 1991; Gary et al., 1993). These are the same kind of ions that, at arrival at comet 67P/CG, caused the so-called singing (Richter et al., 2015), but in a low-density and low-magnetic field environment.

\section{Propagated solar wind}

As there is no upstream solar wind monitor at comet 67P/CG, and changes in solar wind properties are important with respect to the interactions around the comet, two solar wind propagation models are used: Tao et al. (2005) model the solar wind as an ideal MHD fluid; whereas based on Opitz et al. $(2009,2010)$ a ballistic propagation was carried out. The Opitz-Dósa model tries to find the Parker-spiral connecting Rosetta to the Sun, based on ACE velocity measurements, assuming that the state of the Sun and solar wind velocity at a certain Carrington longitude is constant over half a solar rotation. Through checking a range of possible Carrington longitudes as the origin of the plasma measured by ACE, the longitude-velocity pair which results in the least error is chosen. Both methods ignore the latitudinal extension of Rosetta $\left(7^{\circ}\right)$ and propagate solar wind only in the ecliptic plane.

In Fig. 4 the propagated solar wind parameters of both models are shown in panels $\mathrm{d}-\mathrm{g}$. There is a difference in some of the parameters: the Tao-model propagates the tangential magnetic field component, mainly in the $y$ direction of the CSEQ coordinate system; whereas the Opitz-

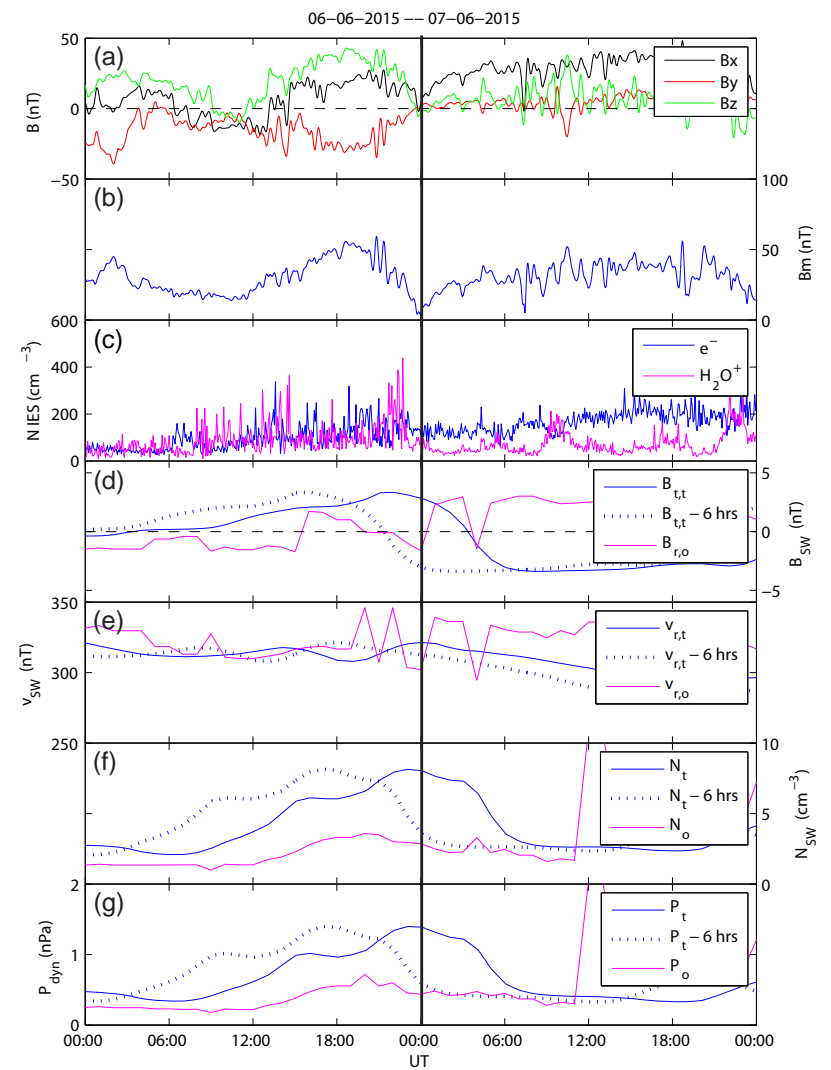

Figure 4. (a) The magnetic field in CESQ coordinates. (b) The magnitude of the magnetic field. (c) The electron and ion velocities deduced from IES. (d) The propagated solar wind magnetic field in cylindrical coordinates. (e) The propagated solar wind velocity in cylindrical coordinates. (f) The propagated solar wind density. (g) The propagated solar wind dynamic pressure.

Dósa-model propagates the radial magnetic field component, mainly in the $x$ direction of CSEQ.

The Tao-model shows that the tangential component of the magnetic field $\boldsymbol{B}_{\mathrm{t}, \mathrm{t}}$ slowly increases in strength and after midnight from 6 to 7 June quickly reverses in sign. With the increase in $\boldsymbol{B}_{\mathrm{t}, \mathrm{t}}$ the density $N_{\mathrm{sw}}$ and dynamic pressure $P_{\mathrm{dyn}}$ also increase. The Opitz-Dósa-model shows that the radial magnetic field, $\boldsymbol{B}_{\mathrm{r}, \mathrm{o}}$, slowly changes from negative to positive, indicating a heliospheric plasma sheet crossing, which would explain the increase in solar wind density. However, this could also be a signature of a corotating interaction region impinging on the comet's plasma surrounding (Edberg et al., 2015a).

As the solar wind velocity does not change during this interval, the increase in dynamic pressure is only created by an increase in ion density, which is clear through the same profiles in panels $f$ and $g$. The solar wind density in the Tao-model increases by a factor of 4 from $N_{\mathrm{sw}} \approx 2$ to $N_{\mathrm{sw}} \approx 8 \mathrm{~cm}^{-3}$ over $\sim 18 \mathrm{~h}$. The Opitz-Dósa-model shows a lesser increase of a factor $\sim 2$. 
As the modelling of the solar wind propagation cannot be perfect, in Fig. 4 the Tao parameters have also been plotted, shifted by $-6 \mathrm{~h}$ as dotted lines. The shift improves the correspondence with the Opitz-Dósa-model and with what is observed at Rosetta. This shows that with the increase of the density and the dynamic pressure, the magnetic field strength measured by Rosetta increases, as should be expected. The Opitz-Dósa-model has a maximum in between the non-shifted and shifted maxima of the Tao-model. Indeed, in general the magnetic field strength in panel $b$ follows the dotted curves in panels $\mathrm{f}$ and $\mathrm{g}$ rather well. Interestingly, after shifting the Tao-model by $-6 \mathrm{~h}$ the change in $\boldsymbol{B}_{\mathrm{t}, \mathrm{t}}$ occurs near the change in $\boldsymbol{B}_{\mathrm{r}, \mathrm{o}}$.

The factor 8 increase in solar wind density could have a significant influence in ionization of the outflowing gas if electron/ion-neutral collisions are important. The creation of a diamagnetic cavity (Glassmeier et al., 2015; Götz et al., 2015) shows that at the location of Rosetta collisions are indeed important. The increased counts, energy and density in the IES and ICA data occur during the shifted increase in solar wind density.

\section{Pile-up and draping}

With the increase in plasma density and magnetic field strength, generated by the increased solar wind dynamic pressure and density, the magnetic field is expected to get more piled-up, as observed, and possibly more draped. For the whole interval the clock $(\xi)$ and cone $(\psi)$ angle of the magnetic field is calculated:

$$
\begin{aligned}
\xi & =\tan ^{-1}\left(\frac{B_{z}}{B_{y}}\right), \\
\psi & =\tan ^{-1}\left(\frac{B_{x}}{\sqrt{B_{y}^{2}+B_{z}^{2}}}\right),
\end{aligned}
$$

the result of which is shown in Fig. 5a. Before the increase in density at $\sim 16: 00$ UT there are variations in both angles, the clock angle $\xi$ rotates from $\sim 180$ to $\sim 90^{\circ}$ then back to $\sim-100^{\circ}$ and back again to $\sim 140^{\circ}$. As the mass loading starts and $\boldsymbol{B}_{\mathrm{m}}$ increases the clock angle $\xi$ remains constant. The cone angle $\psi$ varies also from $\sim 0^{\circ}$ (i.e. in positive $X$ axis direction, towards the Sun) with slight variations in phase with the clock angle $\xi$, moving slightly away from the $x$ direction up to $\psi \approx-45^{\circ}$ and then returns to remain constant at $\psi \approx 20^{\circ}$ during the interval of increased density and magnetic field strength.

When the field strength starts to decrease at $\sim 21: 00 \mathrm{UT}$, and reaches a very low value, $\boldsymbol{B}_{\mathrm{m}} \approx 4 \mathrm{nT}$ around midnight, the cone angle $\psi$ slowly increases to $\sim 85^{\circ}$, i.e. far away from the $x$ direction, whereas the clock angle $\xi$ varies strongly because of the large oscillations in the magnetic field components, the largest of which are also visible in the cone angle.
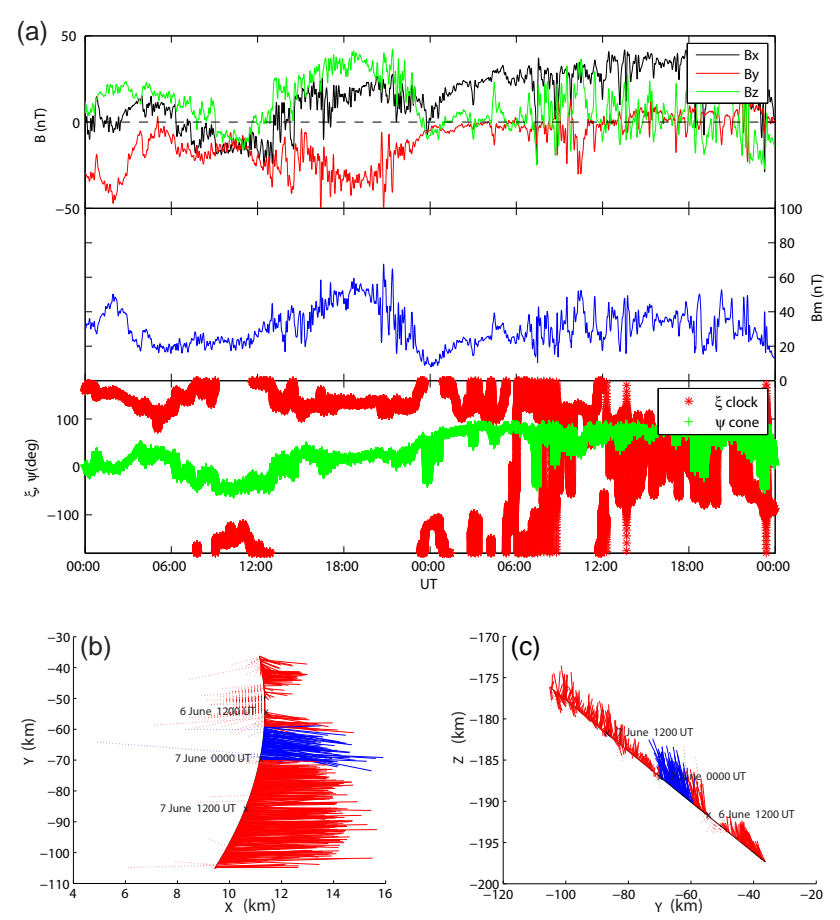

Figure 5. Panel (a) from top to bottom: the magnetic field components, the total magnetic field strength and the clock $\xi$ and cone $\psi$ angle of the magnetic field. Panel (b): the magnetic field plotted along the spacecraft orbit, with 5 min resolution, in the CSEQ $X-Y$ plane. The blue region depicts the interval of compressed magnetic field. The dotted lines show where $B_{\mathrm{X}}<0$. Panel (c): same as panel (b) but in the CSEQ $Y-Z$ plane. The axes in panel (b) and panel (c) have different scales for better visibility.

As there is neither undisturbed solar wind data, nor real undisturbed field around the comet, the draping analysis as proposed by Israelevich et al. (1994) and applied to comet 1P/Halley (see also Delva et al., 2014; Volwerk et al., 2014) cannot be applied. However, the magnetic field direction and behaviour can be looked at in hedgehog-plots, as in Fig. $5 \mathrm{~b}$ and $\mathrm{c}$ for the magnetic field vectors in the $X-Y$ and $Y-Z$ plane along the orbit of the spacecraft. It is clear from the data in Fig. 5a, that on 7 June $B_{x}$ is the main magnetic component, on 6 June before and during the increased density period $B_{y}$ and $B_{z}$ dominate. In the hedgehog-plots the period of the magnetic pile-up is shown in blue, showing that the direction of the field remains constant. Also, it is clear from both the cone and clock angles and the hedgehog-plots that the main rotations of the magnetic field take place before the increase in density, whereas the blue-coloured region and the later data show a rather stable, slightly increasing, largescale magnetic field, only disturbed by the strong oscillations after 7 June 06:00 UT.

The compression of the induced magnetosphere increases the magnetic field strength $\boldsymbol{B}_{\mathrm{m}}$ without a significant change in direction shown by $\xi$ and $\psi$. There are small rotations of 
the field, after the pile-up but the magnetic field pattern does not seem to show any significant change in draping direction.

Before the massloading, between 06:18 and 13:08 UT, the $X$ component of the magnetic field has reversed, from positive to negative.

Magnetic field rotations in the pile-up region of comets are of interest, as they are usually the "memory" of the magnetic field of the solar wind conditions earlier. At Comet 1P/Halley a large set of nested draping regions were found (Raeder et al., 1987). These oppositely directed magnetic fields have to be separated by current sheets and bring the possibility of magnetic reconnection in the cometary coma (see e.g. Verigin et al., 1987; Kirsch et al., 1989, 1990).

The rotation of the magnetic field, as shown in Fig. 5 does not show up clearly in the propagated solar wind data. There is a short change of sign in $\boldsymbol{B}_{\mathrm{t}, \mathrm{t}}$ of the propagated solar wind data between $\sim 00: 00$ and $\sim 02: 30$ UT on 6 June. The minimum field is only $B_{\mathrm{t}, \mathrm{t}, \min } \approx-0.5 \mathrm{nT}$. Neither does the propagated radial $\boldsymbol{B}_{\mathrm{r}, \mathrm{o}}$ component show a field reversal signature.

The boundaries of this $\sim 7 \mathrm{~h}$ region are studied in more detail, and a zoom in on the intervals 05:50-06:50 and 12:4013:40 UT is shown in Fig. 6. The magnetic field data and the ion velocity have been transformed to the minimum variance coordinates system (MVA, Sonnerup and Scheible, 1998, indicated by subscripts min, int and max) derived from the magnetic field, and the red dashed line is the low-pass filtered data with a shortest period of $10 \mathrm{~min}$. Clearly, there is a lot of wave activity in the data. There is little change in the ion velocity direction over the two rotations of the field, only in rotation 1 the $v_{\max }$ seems to change direction after the $\boldsymbol{B}_{\max }$ has changed sign, i.e. moved to the other side of a current sheet. Although in principle this could be a signature of component reconnection, the plasma data are too sparse to draw such a conclusion.

Using the low-pass filtered data (periods longer than $10 \mathrm{~min}$ ), the field changes by $\Delta \boldsymbol{B}_{\max } \approx 21 \mathrm{nT}$ over a timespan of $11 \mathrm{~min}$. With a spacecraft velocity of $v_{\mathrm{sc}} \sim 1 \mathrm{~m} \mathrm{~s}^{-1}$, assuming the rotations convect over Rosetta with this velocity, making $\Delta L \approx 660 \mathrm{~m}$, and using Ampère's law,

$\nabla \times \mathbf{B}=\mu_{0} \mathbf{J}$,

to calculate the current density (neglecting the displacement current):

$\frac{\Delta B}{\Delta L} \approx \mu_{0} J \Rightarrow J \approx 25 \mu \mathrm{Am}^{-2}$.

For the second rotation the field change is $\Delta \boldsymbol{B}_{\max } \approx 35$ over a time span of $7 \mathrm{~min}$, which leads to a current density of $J \approx 66 \mu \mathrm{A} \mathrm{m}^{-2}$. Because of the assumed slow convection velocity $\Delta L$ remains small, an upper limit for $\Delta L$ can be found under the assumption of frozen in fields and a convection velocity of $\sim 10 \mathrm{~km} \mathrm{~s}^{-1}$, which would significantly decrease the current density by a factor $\sim 10^{4}$.
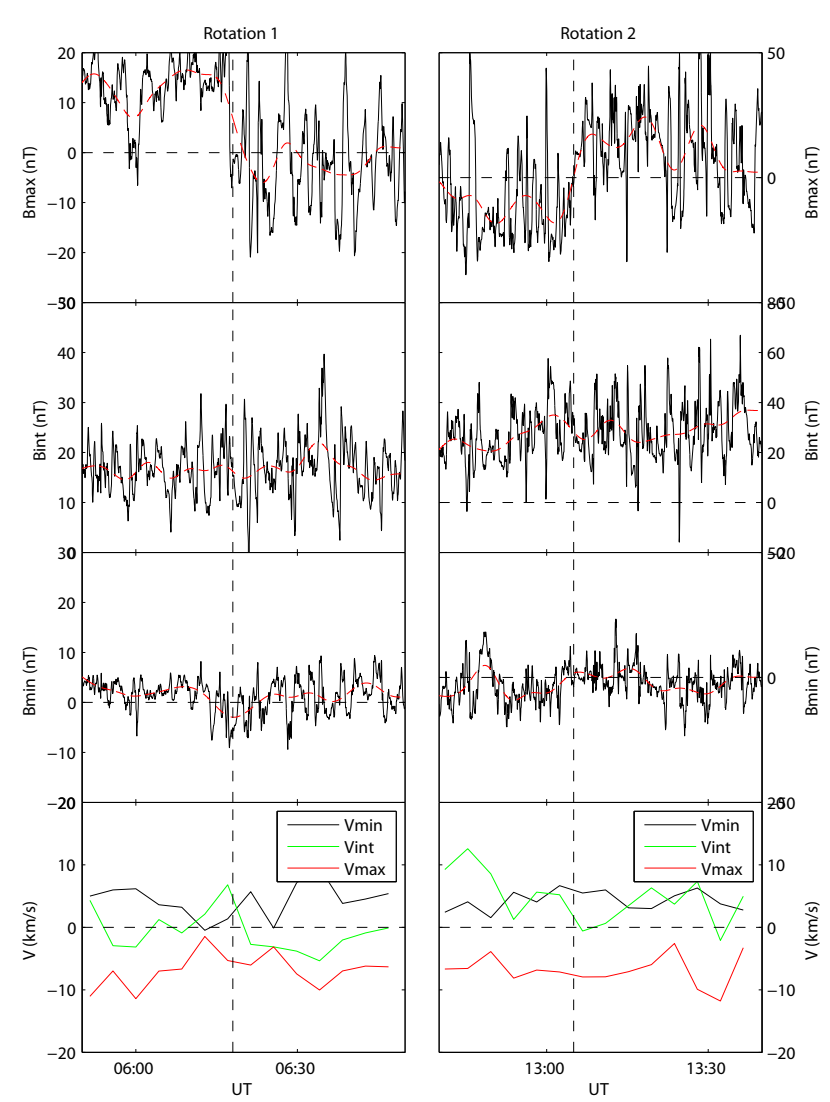

Figure 6. Zoom in on the two $B$-field rotations 1 (left) and 2 (right). Each column showing the magnetic field data in MVA coordinates: $\boldsymbol{B}_{\text {max }}, \boldsymbol{B}_{\text {int }}$ and $\boldsymbol{B}_{\text {min }}$, the black lines show the data and the red lines show the low-pass filtered data. The bottom panel shows the IES ion velocities in MVA coordinates. The vertical dashed line shows the $\boldsymbol{B}_{\max }=0$ crossing.

\section{Crossing from 6 to 7 June: mirror-mode waves}

Pick-up of freshly ionized ions into a streaming magnetoplasma leads to the creation of a ring/ring-beam distribution in velocity space, which is unstable (see e.g. Hasegawa, 1969; Tsurutani et al., 1982; Gary, 1991; Gary et al., 1993). Depending on the plasma- $\beta$ this can lead to either ion cyclotron waves (low- $\beta$ ) or mirror-mode (MM) waves (high- $\beta$ ). In the case of comet $67 \mathrm{P} / \mathrm{CG}$, the plasma- $\beta$ is high and thus MM waves are expected. They were also observed e.g. at comet 1P/Halley (see e.g. Glassmeier and Neubauer, 1993; Schmid et al., 2014; Volwerk et al., 2014). The instability criterion for MM waves is given by:

$$
1+\beta_{\text {perp }}\left(1-\frac{T_{\text {perp }}}{T_{\mid}}\right)<1,
$$

where $T_{\text {perp }}$ and $T_{\mid}$are the ion-temperatures perpendicular and parallel to the background magnetic field and $\beta_{\text {perp }}$ is the perpendicular plasma- $\beta$ determined only using $T_{\text {perp }}$. The MM wave behaves in such a way that the perpendicular pres- 

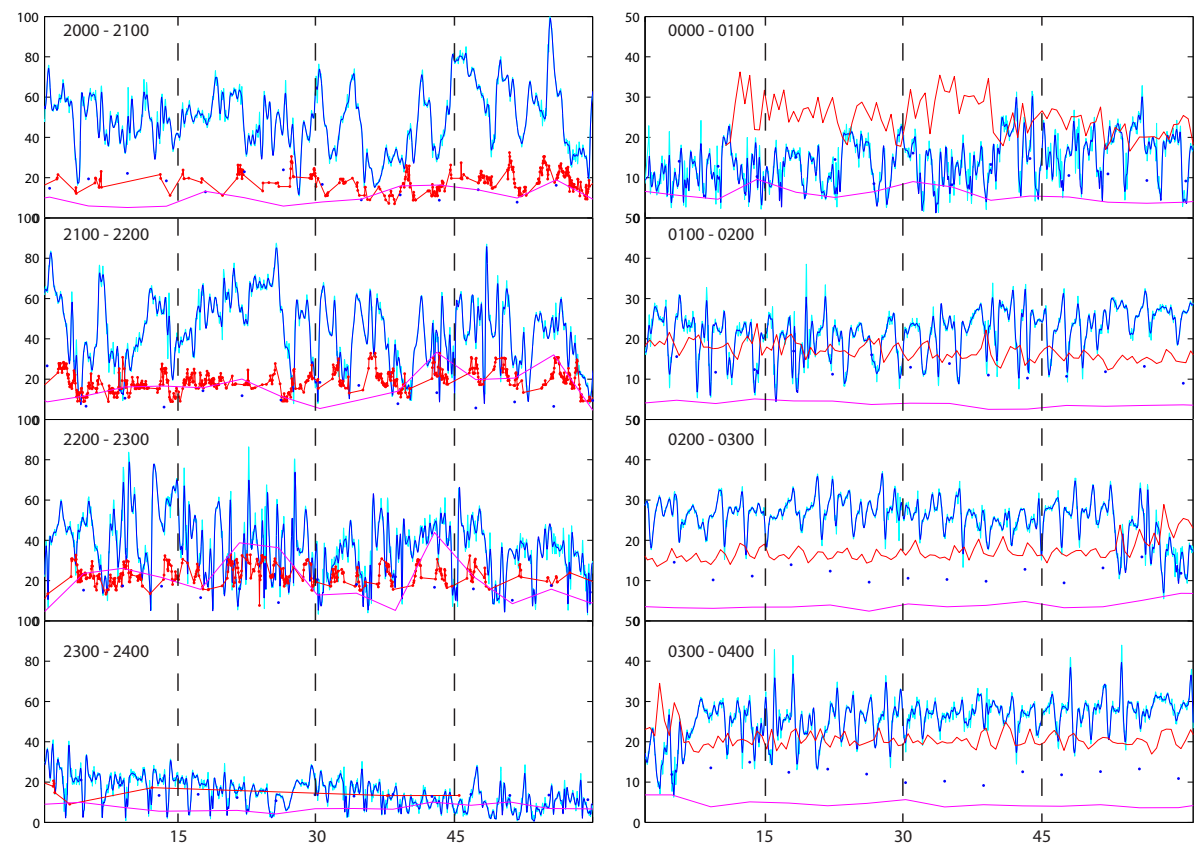

Figure 7. Eight $1 \mathrm{~h}$ intervals of the magnetic field strength $\boldsymbol{B}_{\mathrm{m}}$, left column before midnight of 6 until 7 June and right column after midnight. The cyan line is $\boldsymbol{B}_{\mathrm{m}}$ and the blue is the low-pass filtered field. The magenta line is the IES ion density and the blue dotted line is the IES electron density, the red line and dots are the MIP electron density in the left panels and the LAP P1 current in the right panels. Because of the various different quantities plotted in the panels there are no labels on the $y$ axis.

sure $p_{\text {perp }}$ of the plasma is in anti-phase with the magnetic pressure $p_{B}$, while the total pressure remains constant.

On 7 June the ion density returned to pre-event values, the magnetic activity, however, remains. To study the difference in the 4 hours before and after midnight, the magnetic field and plasma data are plotted in Fig. 7.

It is clear from the panels in Fig. 7 that during the last $4 \mathrm{~h}$ of 6 June (left panels) the MIP electron density variations (red dots) seem to be in phase with the low frequency variations of the total magnetic field. After 6 June $\sim 23: 00$ UT there is no MIP density available anymore and after 7 June $\sim$ 00:10 UT LAP P1 currents are available as a proxy for the plasma density. Over the first $4 \mathrm{~h}$ of 7 June, Fig. 7 right panels, there often seems to be an anti-correlation between the total magnetic field $\boldsymbol{B}_{\mathrm{m}}$ and the LAP P1 current.

Starting at 6 June around $\sim$ 23:00 UT quasi-periodic dips occur in the magnetic field strength, some of which seem to be anti-correlated with the LAP P1 current. This could imply that the freshly mass-loaded magnetospheric magnetic field is mirror-mode unstable (see e.g. Hasegawa, 1969; Glassmeier et al., 1993; Tsurutani et al., 1999; Schmid et al., 2014; Volwerk et al., 2014). As the resolution of the plasma data is too low to check the pressure balance over the MM structures, the magnetic-field-only method by Lucek et al. (1999) is used to investigate the data for MM waves. These waves are expected to have strong magnetic field variations, $\Delta B / B$, and they are non-propagating structures, only convected by the streaming magnetoplasma in which they are embedded.
This means that in an MVA the minimum variance direction should be perpendicular to the background magnetic field and the maximum variance direction along the background magnetic field. A study by Price et al. (1986) showed that the angle between maximum variance direction and background field was smaller than $30^{\circ}$.

MVA is applied to the RPC-MAG data over a sliding window, and the angles $\theta$ of the minimum variance and $\phi$ of the maximum variance directions with respect to the low-pass filtered (longer than $10 \mathrm{~min}$ ) background magnetic field are determined, as well as the relative amplitude $\Delta B / B$ defined as twice the variation: $\Delta B / B=2\left(B-B_{\mathrm{bg}}\right) / B_{\mathrm{bg}}$. In order for MM identification, the structures have to fulfill the following criteria: $\theta \geq 80^{\circ}, \phi \leq 20^{\circ}$ and $\Delta B / B \geq 1$. In Fig. 8 an 8 hour interval is shown, on which the MM determination has been performed. Panel a shows the IES electron (blue) and ion (cyan) density, in panels b-e the $1 \mathrm{~s}$ resolution MAG data are shown in black with the low-pass filtered data overplotted in red. Panel $\mathrm{f}$ shows $\Delta B / B$ and panel $\mathrm{g}$ the angles $\theta$ (green) and $\phi$ (red). There are regions where the above criteria are fulfilled, but it is difficult to see in this figure. Therefore, short intervals will be analysed separately below.

A zoom-in on two 10-min intervals of Fig. 8, and adding the density data of either MIP or LAP is shown in Fig. 9. In the first interval 22:30-22:40 UT there are short periods where the criteria are almost fulfilled, the maximum variance angle $\phi$ is rather large. Unfortunately, the electron density estimated by MIP is unavailable when the plasma frequency 


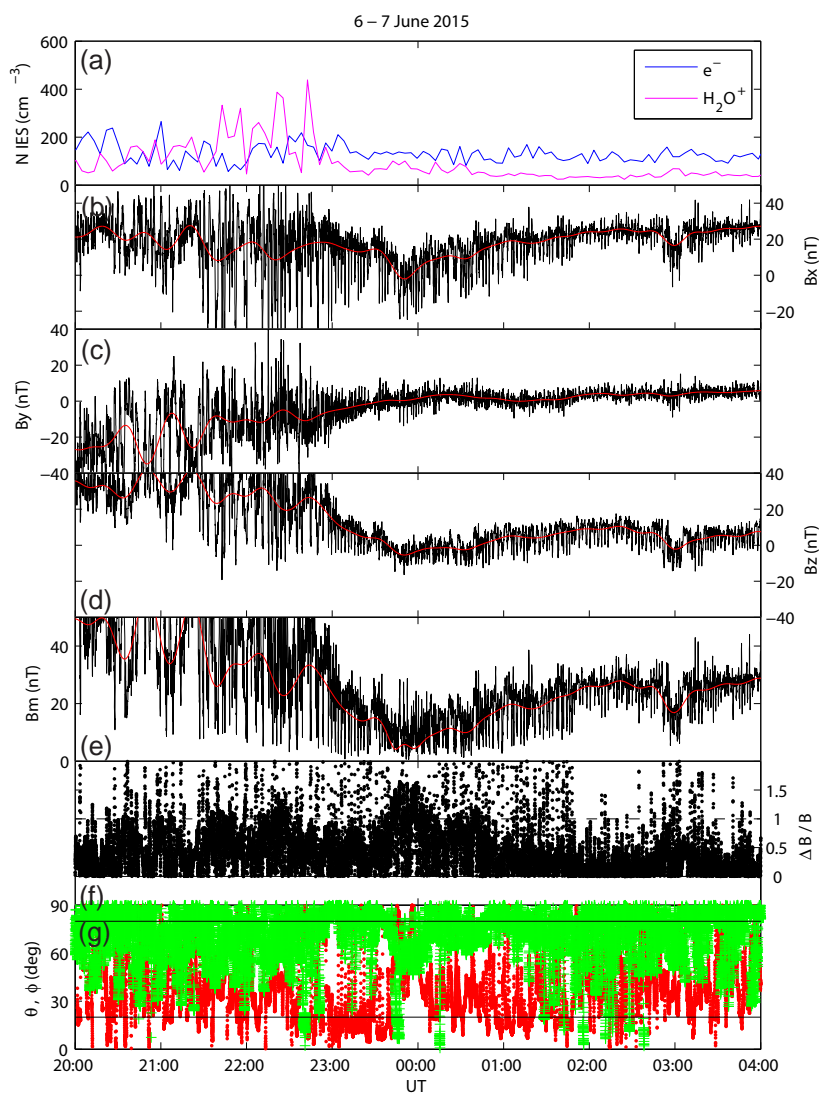

Figure 8. (a) The IES electron and ion density. (b-e) The magnetic field components and field strength in black and the band-pass filtered data in red. (f) The $\Delta B / B$ from the MM identification procedure. (g) Angles $\theta$ (green) and $\phi$ (red) between the background magnetic field the minimum variance and the maximum variance direction respectively.

is out of the frequency range of the instrument, or when the electron density is small enough and the electron temperature high enough for the Debye length to be much larger than the instrument emitter-receiver length scale. This makes it difficult to find a correlation between $\boldsymbol{B}_{\mathrm{m}}$ and $N_{e}$ for the whole time series. Before 22:35 UT, when $\theta>80^{\circ}$ it is difficult to interpret the electron density and thus the inset panel zooms in once more on the interval 22:31-22:32:30 UT. There it is clear that the MIP electron density is in anti-phase with the non-filtered magnetic field strength (cyan).

During the second interval of 01:10-01:20 UT, the LAP P1 current acts as a proxy for the plasma density. In this case it is clear in Fig. 9 right panels that $\theta$ and $\phi$ are close to the MM criteria. The two strong dips in $\boldsymbol{B}_{\mathrm{m}}$ in the first $5 \mathrm{~min}$ show that as the field strength decreases the current increases.

This means that the mass-loading of the induced magnetosphere of comet $67 \mathrm{P} / \mathrm{CG}$ created an unstable ion population through pick-up (a ring/ring-beam distribution), which relaxes through the generation of mirror-mode waves. Indeed, such a distribution was posited above when looking at the

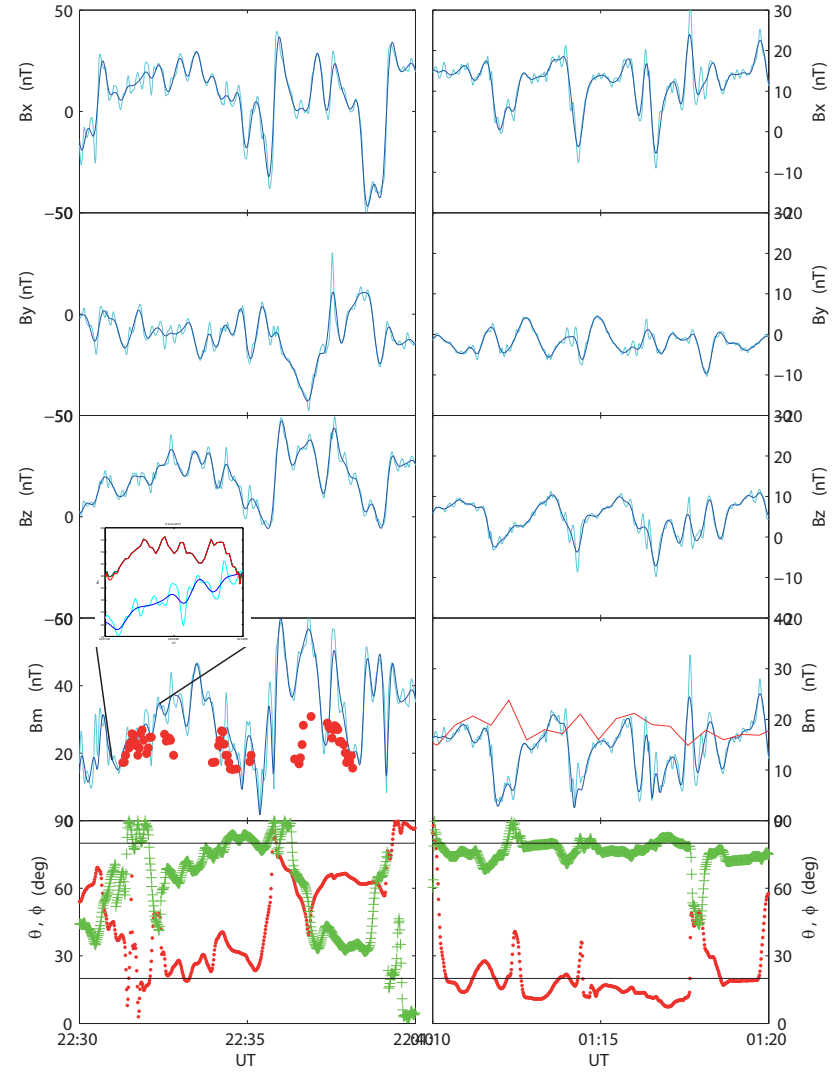

Figure 9. Zoom in on two intervals with different mirror mode waves. From top to bottom the magnetic field components; the magnetic field strength with overplotted in red the MIP electron density (left) or the LAP $\mathrm{P}^{\prime}$ current. The bottom panels shows the angles $\theta$ and $\phi$ from the MM determination procedure. The inset in the left column shows a blow up of a short interval which shows that the MIP electron density is in anti-phase with the non-filtered $\boldsymbol{B}_{\mathrm{m}}$ data.

ion velocity direction with respect to the background magnetic field. The question whether such a distribution is able to develop in the cometosheath under the above conditions is addressed in the discussion section below.

On 7 June, the MM structures have, on average, a timescale of $100 \leq T_{\mathrm{mm}} \leq 150 \mathrm{~s}$, which will be compared to a characteristic length scale of pick-up ions, being the Larmor radius. Assuming that the newly formed ions are picked up with the local (decelerated) solar wind velocity $v_{\mathrm{SW}}$, the gyro frequency $\omega_{c, i}$ and radius $\rho_{c, i}$ are given by

$$
\begin{aligned}
\omega_{c, i} & =\frac{q_{i} B}{m_{i}}, \\
\rho_{c, i} & =\frac{v_{\text {perp }}}{\omega_{c, i}},
\end{aligned}
$$

also assuming that $v_{\text {perp }}=v_{\mathrm{SW}}$ and that the structures are transported with $v_{\mathrm{SW}}$ over the spacecraft and have a size of 

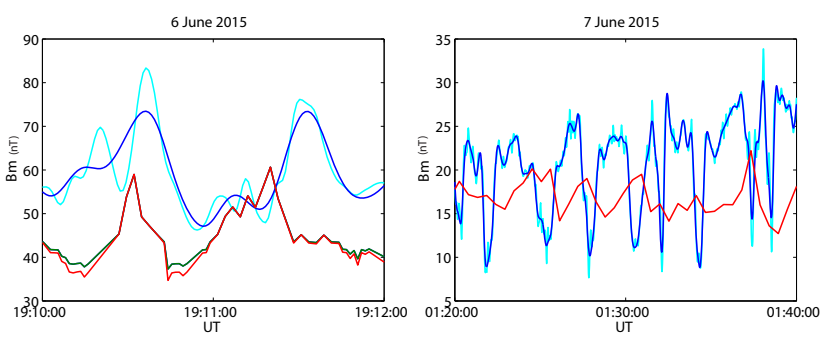

Figure 10. Left: zoom in on interval 19:10-19:12 UT, the MIP electron density is following $\boldsymbol{B}_{\mathrm{m}}$, fil (blue) in the first minute. Just after 19:11 UT the MIP is almost in anti-phase with the non-filtered $\boldsymbol{B}_{\mathrm{m}}$ (cyan) for about 30 s. Right: zoom in on interval 01:21-01:40 UT showing that the LAP P1 current (red) is in anti-phase with $\boldsymbol{B}_{\mathrm{m}}$.

$\alpha \rho_{c, i}$ the timescale is given by

$T_{\mathrm{mm}}=\frac{\alpha \rho_{c, i}}{v_{\mathrm{SW}}}=\frac{\alpha}{\omega_{c, i}}$.

This means that for these assumptions the solar wind velocity drops out of the equations and the crossing time is given by known and measured quantities. For water ions at a magnetic field strength of $\boldsymbol{B}_{\mathrm{m}} \approx 20 \mathrm{nT}$ this leads to $T_{\mathrm{mm}} \approx$ $9 \alpha \mathrm{s}$. With the measured $T_{\mathrm{mm}}$ mentioned above this leads to $11 \leq \alpha \leq 16$, which is similar to what was found by Tsurutani et al. (1999) at comet 21P/Giacobini-Zinner, $\alpha_{\mathrm{GZ}} \approx 12$, but much larger than what was found by Schmid et al. (2014) at comet $1 \mathrm{P} /$ Halley, $\alpha_{\mathrm{H}} \approx 1-2$. Taking the ion velocity as measured by IES, the Larmor radius for water ions becomes $\rho_{\mathrm{H}_{2} \mathrm{O}, \mathrm{i}} \approx 280 \mathrm{~km}$.

For the interval 22:30-22:40 UT it is clear that the size of the alleged MM structure is much smaller than in the later interval discussed above. An estimate from the inset panel in Fig. 9 shows that the MM structures have a timespan of $\sim 10 \mathrm{~s}$. The field strength is slightly higher at $\boldsymbol{B}_{\mathrm{m}} \approx 25 \mathrm{nT}$, which means from Eq. (8) that $T_{\mathrm{mm}} \approx 7 \alpha$. This means that $\alpha \approx 1.4$, which is more in line with the results of Schmid et al. (2014) for freshly picked-up water ions near comet 1P/Halley.

Similar MM intervals can be found earlier, 19:1019:12 UT, as shown in Fig. 10 left panel. The timespan is again $T_{\mathrm{mm}} \sim 10 \mathrm{~s}$, with a field strength $\boldsymbol{B}_{\mathrm{m}} \approx 55 \mathrm{nT}$ this leads to $\alpha \approx 2.7$. Again, at the beginning of the interval, the MIP electron density seems to follow the filtered magnetic field strength (blue). In the middle, just after 19:11 UT, the electron density is almost in anti-phase with the non-filtered magnetic field strength (cyan). And MMs can also be found later, 01:21-01:40 UT, as shown in Fig. 10 right panel. Here the structures are again larger, on the order of $T_{\mathrm{mm}} \approx 100 \mathrm{~s}$ and $\alpha \approx 11$ with the LAP P1 current in anti-phase with the magnetic field strength.
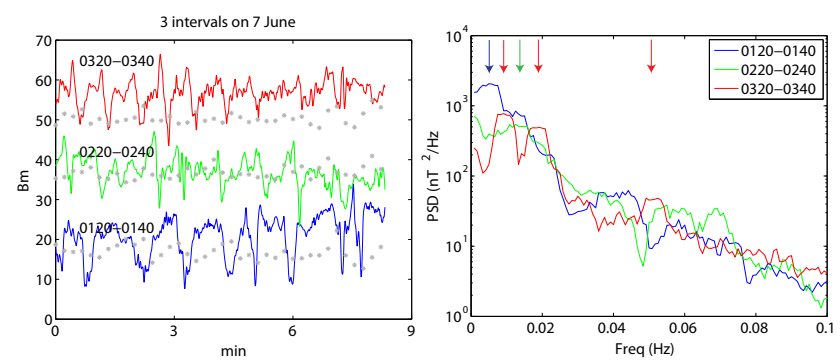

Figure 11. Left panel: three $20 \mathrm{~min}$ intervals of the magnetic field strength $\boldsymbol{B}_{\mathrm{m}}$ data, shifted to enhance visibility - blue 01:2001:40 UT, green 02:20-20:40 UT, red 03:20-03:40UT. The grey stars show the LAP P1 current. Right panel: the Fourier power spectra for the three intervals. The coloured arrows at the top mark the peaks discussed in the text.

\section{Change of MM shape}

A closer look at Fig. 7 right panel shows that the structures, identified as mirror-mode waves, are changing in shape. Indeed, in the top panel the structures seem to be mainly dips in the magnetic field strength, $\boldsymbol{B}_{\mathrm{m}}$, but at later times the structures seem to become asymmetric. A zoom-in on three intervals of $20 \mathrm{~min}$ is shown in Fig. 11; the data are shifted along the $y$ axis in order to make the difference between them more visible. The LAP P1 current is shown as grey asterisks overplotted on each interval. The three intervals are different in behaviour: the first interval 01:20-01:40 UT (blue) shows mainly strong dips in $\boldsymbol{B}_{\mathrm{m}}$; the second interval 02:20-02:40 UT (green) shows strong asymmetric dips in $\boldsymbol{B}_{\mathrm{m}}$ and a large variety in structure sizes; the third interval 03:20-03:40 UT (red) shows in the beginning deformation of the waves, strong periodic peaks with moving peaks super-imposed.

Spectral analysis is performed on these three intervals. It is clear from Fig. 11 right panel, that the three intervals have different spectral content: the first interval (blue) has a peak at $f \approx 6 \mathrm{mHz}$ and a minor peak at $f \approx 13 \mathrm{mHz}$, the second interval (green) shows a plateau-like structure around $f \approx$ $10 \mathrm{mHz}$; the third interval (red) shows a clear double peaked structure at $f \approx 9$ and $f \approx 19 \mathrm{mHz}$ with a minor peak at $f \approx$ $51 \mathrm{mHz}$, which explains the beat-mode that can be seen in the red trace in Fig. 11 left panel. It is not very clear from the LAP P1 current to deduce that these structures are mirrormodes, although the Lucek method indicates that they are.

\section{$7 \quad$ Discussion and conclusions}

For the first time in space research history a spacecraft is following a comet along its orbit from pre- to post-perihelion, entering regions around the comet that up to now had not been accessed. Also the outgassing of comet 67P/CG at arrival in August 2014 was at a much lower level than for any other mission. During the period discussed in this paper the 
outgassing rate is around $10^{27}$ molecules $\mathrm{s}^{-1}$, which is several orders of magnitude smaller than at comets 27P/GriggSkjellerup (Neubauer et al., 1993) or 1P/Halley (Reinhard, 1986). This means that the interaction of the solar wind with the outgassing comet is different, which was clearly illustrated through the discovery of the "singing comet" by Richter et al. (2015), an unexpected plasma instability created by the not-yet-magnetized freshly produced ions near the comet. This is the context in which the results of this paper should be interpreted: measurements much closer to a cometary nucleus than ever before, with low outgassing rate and a very slowly moving spacecraft relative to the nucleus.

The data from RPC MAG have been calibrated, however Richter et al. (2015) state that: "The short boom length implies that the spacecraft is heavily contaminating the magnetic field measurements. At this stage of the investigation it was not possible to completely remove these quasi-static spacecraft bias fields from the measured magnetic field values.". In this current paper, the observations of the diamagnetic cavity (Glassmeier et al., 2015; Götz et al., 2015) have been used to obtain values for non-corrected bias fields originating from the spacecraft. Assuming that the diamagnetic cavity should be field-free (see e.g. Ip and Axford, 1987), the measured fields in the cavity have been subtracted from the data. This leads to a greatly improved determination of the mirror mode waves using the magnetic-field-only technique (Lucek et al., 1999), as the examples shown in Fig. 9 would not have been selected without bias-field offset correction.

The mass loading of the induced magnetosphere of comet 67P/CG, as indicated by the Rosetta ROSINA-COPS and RPC plasma instruments showed an interesting behaviour on the 2 days discussed in this paper (6-7 June 2015). At the beginning of 6 June COPS shows an increase in neutral density (first dashed line in Fig. 2 near 02:45 UT), but the RPC plasma instruments do not show any significant response. With the second strong increase in neutral density near 08:30 UT, there is some increase in energy in the ions and the IES electron and ion density slowly increase. However, after the third maximum in COPS near 15:45 UT, both IES and ICA start to show a significant increase in both counts and energy of the ions. Indeed, with every next neutral gas maximum there is a slow increase in counts and energy.

The observed variations in the solar wind parameters, such as directional changes and increase in dynamic pressure, in both solar wind propagation models used in this paper, led to several interesting phenomena:

- Before the increased density and the pile-up region there was a rotation of the magnetic field. This is probably related to changes in the field direction of the solar wind magnetic field, generating nested draped fields around the comet.

- Depending on the assumption how fast Rosetta crosses this structure the current densities in the current sheet are tens or $\mu \mathrm{Am}^{-2}$ or several $\mathrm{nA} \mathrm{m}{ }^{-2}$.
- There is increased ionization and energization of gas from the cometary nucleus in both IES and ICA.

- The magnetic field strength increased by a factor of $>3$ up to $\sim 60 \mathrm{nT}$, increasing the magnetic pressure. With the ion density on the order of $100 \mathrm{~cm}^{-3}$ and the ion temperature a few $10^{5} \mathrm{~K}$, this means that the plasma beta $\beta \sim 10$.

- The newly created ions are accelerated by the motional electric field, however, the effect only becomes apparent after the pile-up region is exited by the spacecraft.

- In the pile-up region there is evidence for mirror-mode structures, generated by the newly created ions, with a size between one and three water-ion gyro radii.

- Outside the pile-up region there are clear signatures of mirror-mode waves, with a much larger size of 10 to 16 water-ion gyro radii.

- Outside the pile-up region there is a development of the mirror-mode structures, where at later times there are three dominant frequencies present, which leads to strong deformation of the mirror-mode waves signature in the MAG data.

The above results leave a few points to discuss which will be addressed below.

\section{- Nested draping:}

The change in direction of the magnetic field as observed in the Rosetta data does not show up clearly in the propagated solar wind magnetic field. The Taotangential field seems to go negative for a short period in the non-shifted data in Fig. 4 at the beginning of 6 June. The Opitz-Dósa-radial magnetic field basically shows a heliospheric current sheet crossing. Because of the draping and hanging-up of the magnetic field around the comet, it is difficult to find a one-to-one correlation between the solar wind field signatures and the draped field signatures. The layer of differently directed field at Rosetta may be the result of an older interval outside that presented in the figure. The difference in field strength can be explained through the compression by the solar wind pressure.

- Changes in the magnetic pile-up region:

Rosetta is located well inside the MPR of comet 67P/CG, which is clear from the high magnetic field strength measured by MAG, $\boldsymbol{B}_{\mathrm{m}} \geq 20 \mathrm{nT}$ and the expected solar wind magnetic field strength $\boldsymbol{B}_{\mathrm{sw}} \approx 2 \mathrm{nT}$. The build-up of a magnetic pile-up region is related to pressure balance from the draped magnetic field pushing outward and the solar wind dynamic pressure pushing inward towards the comet. With the increase in solar wind pressure, this balance is disturbed and the 
field gets compressed more. This is what is observed in the MAG data, where the 4-fold increase in dynamic pressure leads to a magnetic field strength increase by a factor $\sim 2.5$ from $\sim 20$ to $\sim 55 \mathrm{nT}$. This agrees well with the expected increase, which would be $\sqrt{P_{\mathrm{dyn}, \max } / P_{\mathrm{dyn}, \min }}$.

- Ionization increase:

Looking at a longer data set of the IES ion energy spectra, it is clear that this increase in counts and energy of the ions is limited to a period of $\leq 18 \mathrm{~h}$, which corresponds to the increased solar wind dynamic pressure, which is caused by an increase of the solar wind density. This means that an enhanced number of solar wind electrons is also entering the pile-up region, which increases collisions and ionization as observed by RPC. After this period the IES densities follow the periodicity in the COPS neutral density, indicating that the increased ionization was indeed generated by the higher solar wind density.

- Ring/ring-beam distribution:

A ring/ring-beam distribution is assumed necessary for the generation of the mirror mode waves. However, do the pick-up ions have enough time to develop such a distribution? The IES ion velocity in the increased pileup region shows that the ions are basically moving perpendicular to the magnetic field. With a magnetic field strength between 20 and $55 \mathrm{nT}$ and a velocity of $\sim$ $12 \mathrm{~km} \mathrm{~s}^{-1}$ the gyro frequency is $0.1 \leq \omega_{c, i} \leq 0.25 \mathrm{~s}^{-1}$ and the gyro radius is $50 \leq \rho_{c, i} \leq 120 \mathrm{~km}$. In order for a ring distribution to occur, the collision frequency must be much smaller than the gyro frequency. The collisional time is given by $\tau_{\text {coll }}=\left(n \sigma_{i} v\right)^{-1}$, where $\sigma_{i} \propto 10^{-16} \mathrm{~m}^{2}$ is a typical ion-neutral collisional crosssection (A'Hearn and Festou, 1990). Using typical values $n \geq 10^{6} \mathrm{~cm}^{-3}$ and $v=10 \mathrm{~km} \mathrm{~s}^{-1}$ this leads to $\tau_{\text {coll }} \approx 10^{\overline{3}}-10^{4}$ s. With a gyro period of $25 \leq \tau_{c, i} \leq$ $60 \mathrm{~s}$ this means there is ample time for the ions to create a ring-beam distribution and the location of Rosetta with respect to the comet, $\sim 225 \mathrm{~km}$ shows that the coma is large enough for full gyrations of the ions with the gyro radii mentioned above.

- Different sizes of MMs:

Within the pile-up region, in the second half of 6 June, at high density, the mirror mode waves are between one and three water gyro radii in size. This is "as expected" from newly created $\mathrm{H}_{2} \mathrm{O}^{+}$, as measured, e.g. at comet 1P/Halley (Schmid et al., 2014). Many hours later, on 7 June, there are much larger MM structures in the MAG data, with a size between 10 and 16 gyro radii. The larger structures could possibly be generated by diffusion of smaller size MMs as described by Hasegawa and Tsurutani (2011):

$$
\lambda(L)=\alpha \rho_{c, i}\left[1+\left(\omega_{c, i} L / 32 u\right)\right],
$$

where the source size has been changed to $\alpha \rho_{c, i}$. Putting in the measured values $(\lambda(L)=14, \alpha=2, u=$ $10 \mathrm{~km} \mathrm{~s}^{-1}$ ) and solving for the diffusion distance $L \sim$ $10^{5} \mathrm{~km}$ shows that the large structures cannot have evolved from diffusion of the small structures in the pile-up region. Thus these large structures find their origin in MMs created further upstream in the comet's coma. Where exactly cannot be determined as the source size $\alpha$ of the MMs further upstream is unknown.

- Structure deformation:

The main ion species discussed in this paper is $\mathrm{H}_{2} \mathrm{O}^{+}$, however, $\mathrm{CO}^{+}$and $\mathrm{CO}_{2}^{+}$were almost equal to that of water. Hässig et al. (2015) showed that the detector signal of the ROSINA instrument for all three species was on average $\sim 2 \times 10^{5}$ particles $(20 \mathrm{~s})^{-1}$, with only variations depending on which side of the comet is facing Rosetta. Assuming that the two main frequencies in the spectrum of the third interval in Fig. 11, with deformed (beating?) MM waves, are related to gyro frequencies of pick-up ions, then the ratio of the frequencies should possibly be related to the ratio of the masses of the ions. The low frequency waves are at $\sim 9$ and $\sim 19 \mathrm{mHz}$, which have a frequency-ratio of $\sim 0.47$, the mass-ratio of water with carbon(di)oxide is $0.6 / 0.41$. The ratios are close, which might suggest that there are indeed different kinds of MMs at the same time. This would ask for an interaction of multiple kinds of MMs in one multi-component plasma, which has not been discussed in the literature.

The Rosetta mission around comet 67P/ChuryumovGerasimenko offers excellent opportunities to investigate processes that have been observed during flybys of other comets. Due to the slow motion of the spacecraft with respect to the comet an in-depth view is obtained of the interaction of the solar wind with the outgassing comet. This paper gives a "short" first discussion of a 2-day interval of the data. With the spacecraft in basically the same location near comet 67P/CG this gave the possibility to study the reaction of the induced magnetosphere with respect to the increased solar wind dynamic pressure. Furthermore, in this way temporal variations in the cometosheath, e.g. the changes in the characteristics of the mirror mode waves were studied. Numerical modelling of the events showed in this paper is underway, as well as theoretical investigations into the various mirror-mode waves in a multi-ion pick-up plasma.

Acknowledgements. Rosetta is an ESA mission with contributions from its Member States and NASA. We acknowledge the staff of CDDP and IC for the use of AMDA and the RPC Quicklook database (provided by a collaboration between the Centre de Données de la Physique des Plasmas, supported by CNRS, CNES, Observatoire de Paris and Université Paul Sabatier, Toulouse and Imperial College London, supported by the UK Science and Technology 
Facilities Council). The work of K.-H. Glassmeier, I. Richter and C. Koenders was financially supported by the German Bundesministerium für Wirtschaft und Energie and the Deutsches Zentrum für Luft- und Raumfahrt under contract 50 QP 1401 for Rosetta. C. Carr and E. Cupido thank the UK space agency for support of the Imperial College RPC team. T. Broiles, J. Burch and K. Mandt acknowledge support by NASA for work on IES through contract \#1345493 with the Jet Propulsion Laboratory, California Institute of Technology. N. J. T. Edberg acknowledges support from the Swedish National Space Board and the Swedish Research Council. A. Opitz and M. Dósa are grateful to Z. Neth for the deep discussions and L. Földy for his computational support. The authors acknowledge the ACE and OMNI databases for solar wind data.

The topical editor, E. Roussos, thanks two anonymous referees for help in evaluating this paper.

\section{References}

A'Hearn, M. F. and Festou, M. C.: The neutral coma, in: Physics and chemistry of comets, edited by Huebner, W. F., pp. 69-112, Springer Verlag, Berlin, Germany, 1990.

Alfvén, H.: On the theory of comet tails, Tellus, 9, 92-96, 1957.

Balsiger, H., Altwegg, K., Bochsler, P., Eberhardt, P., Fischer, J., Graf, S., Jäckel, A., Kopp, E., Langer, U., Mildner, M., Müller, J., Riesen, T., Rubin, M., Scherer, S., Wurz, P., Wüthrich, S., Arijs, E., Delanoye, S., Keyser, J. D., Neefs, E., Nevejans, D., Rème,, H., Aoustin, C., Mazelle, C., Médale, J.-L., Sauvaud, J. A., Berthelier, J.-J., Bertaux, J.-L., Duvet, L., Illiano, J.-M., Fuselier, S. A., Ghielmetti, A. G., Magoncelli, T., Shelley, E. G., Korth, A., Heerlein, K., Lauche, H., Livi, S., Loose, A., Mall, U., B.Wilken, Gliem, F., Fiethe, B., Gombosi, T. I., Block, B., Carignan, G. R., Fisk, L. A., Waite, J. H., Young, D. T., and Wollnik, H.: ROSINA - Rosetta Orbiter Spectrometer for Ion and Neutral Analysis, Space Sci. Rev., 128, 697-712, doi:10.1007/s11214006-8335-3, 2007.

Bendat, J. S. and Piersol, A. G.: Measurement and analysis of random data, John Wiley \& Sons, New York, USA, 1966.

Biermann, L.: Physical processes in comet tails and their relation to solar activity, in: La Physique des Comètes, Louvain, Belgium, pp. 251-262, 1953.

Broiles, T. W., Burch, J. L., Clark, G., Koenders, C., Behar, E., Goldstein, R., Fuselier, S. A., Mandt, K. E., Mokashi, P., and Samara, M.: Rosetta observations of solar wind interaction with the comet 67P/Churyumov-Gerasimenko, Astron. Astrophys., 583, aa26046-15, doi:10.1051/0004-6361/201526046, 2015.

Burch, J. L., Goldstein, R., Cravens, T. E., Gibson, W. C., Lundin, R. N., Pollock, C. J., Winningham, J. D., and Young, D. T.: RPCIES: The Ion and Electron Sensor of the Rosetta Plasma Consortium, Space Sci. Rev., 128, 697-712, doi:10.1007/s11214-0069002-4, 2006.

Carr, C., E.Cupido, Lee, C. G. Y., Balogh, A., Beek, T., Burch, J. L., Dunford, C. N., Eriksson, A. I., Gill, R., Glassmeier, K.H., R. Goldstein, D. L., Lundin, R., Lundin, K., Lybekk, B., Michau, J. L., Musmann, G., Nilsson, H., Pollock, C., Richter, I., and Trotignon, J.: RPC: The Rosetta Plasma Consortium, Space Sci. Rev., 128, 697-712, doi:10.1007/s11214-006-9136-4, 2007.

Cravens, T. E. and Gombosi, T. I.: Cometary magnetospheres: a tutorial, Adv. Space Res., 33, 1968-1976, 2004.
Delva, M., Bertucci, C., Schwingenschuh, K., Volwerk, M., and Romanelli, N.: Magnetic pileup boundary and field draping at Comet Halley, Planet. Space Sci., 96, 125-131, 2014.

Edberg, N. J. T., Eriksson, A. I., Johansson, F., Odelstad, E., Vigren, E., Burch, J. L., Carr, C. M., Cupido, E., Glassmeier, K.-H., Goldstein, R., Halekas, J. S., Henri, P., Koenders, C., Lebreton, J.-P., Mandt, K., Mokashi, P., Nemeth, Z., Nilsson, H., Ramstad, R., Richter, I., and Wieser, G. S.: Solar wind interaction with comet 67P: impacts of corotating interaction regions, J. Geophys. Res., submitted, 2015a.

Edberg, N. J. T., Eriksson, A. I., Odelstad, E., Henri, P., Lebreton, J.-P., Gasc, S., Rubin, M., André, M., Gill, R., Johansson, E. P. G., Johansson, F., Vigren, E., E.Wahlund, J., Carr, C. M., Cupido, E., Glassmeier, K.-H., Goldstein, R., Koenders, C., Mandt, K., Nemeth, Z., Nilsson, H., Richter, I., Stenberg-Wieser, G., Szegö, K., and Volwerk, M.: Spatial distribution of low-energy plasma around comet 67P/CG fromRosetta measurements, Geophys. Res. Lett., 42, 4263-4269, doi:10.1002/2015GL064233, 2015b.

Eriksson, A. I., Boström, R., Gill, R., Åhlén, L., Jansson, S.-E., Wahlund, J.-E., André, M., Mälkki, A., Holtet, J. A., Pedersen, B. L. A., and Blomberg, L. G.: RPC-LAP: The Rosetta Langmuir Probe Instrument, Space Sci. Rev., 128, 729-744, doi:10.1007/s11214-006-9003-3, 2006.

Gary, S. P.: Electromagnetic ion/ion instabilities and their consequences in space plasmas: A review, Space Sci. Rev., 56, $373-$ 415, 1991.

Gary, S. P., Fuselier, S. A., and Anderson, B. J.: Ion anisotropy instabilities in the magnetosheath, J. Geophys. Res., 98, 1481-1488, 1993.

Glassmeier, K.-H. and Neubauer, F. M.: Low-frequency electromagnetic plasma waves at comet P/Grigg-Skjellerup: overview and spectral characteristics, J. Geophys. Res., 98, 20921-20935, 1993.

Glassmeier, K. H., Motschmann, U., Mazalle, C., Neubauer, F. M., Sauer, K., Fuselier, S. A., and Acuña, M. H.: Mirror modes and fast magnetoaucoustic waves near the magnetic pileup boundary of comet P/Halley, J. Geophys. Res., 98, 20955-20964, 1993.

Glassmeier, K.-H., Richter, I., Diedrich, A., Musmann, G., Auster, U., Motschmann, U., Balogh, A., Carr, C., Cupido, E., Coates, A., Rother, M., Schwingenschuh, K., Szegö, K., and Tsurutani, B.: RPC-MAG the flux-gate magnetometer in the ROSETTA plasma consortium, Space Sci. Rev., 128, 649-670, 2007.

Glassmeier, K.-H., Goetz, C., Koenders, C., Altwegg, K., Burch, J., Carr, C., Cupido, E., Eriksson, A., Goldstein, R., Güttler, C., Henri, P., Lebreton, J.-P., Mokashi, P., Motschmann, U., Nemeth, Z., Nilsson, H., Richter, I., Rubin, M., Stoll, B., Szegö, K., Tsurutani, B., Vallat, C., and Volwerk, M.: First Detection of a Diamagnetic Cavity at 67P/Churyumov-Gerasimenko, AGU abstracts 14-18 December 2015, AGU Fall Meeting 2015, San Francisco, CA, USA, 2015.

Götz, C., Glassmeier, K.-H., and More: First Detection of a Diamagnetic Cavity at Comet 67P/Churyumov-Gerasimenko, Ann. Geophys., submitted, 2015.

Gringauz, K. I., Gombosi, T. I., Tátrallyay, M., Verigin, M. I., Remizov, A. P., Richter, A. K., Apáthy, L., Szemerey, I., Dyachkov, A. V., Balakina, O. V., and Nagy, A. F.: Detection of a new "chemical" boundary at comet Halley, Geophys. Res. Lett., 13, $613-616,1986$. 
Hasegawa, A.: Drift mirror instability in the magnetosphere, Phys. Fluids, 12, 2642-2650, 1969.

Hasegawa, A. and Tsurutani, B. T.: Mirror mode expansion in planetary magnetosheaths: Bohm-like diffusion, Phys. Rev. Lett., 107, 245005, 2011.

Hässig, M., Altwegg, K., Balsiger, H., Bar-Nun, A., Berthelier, J. J., Bieler, A., Bochsler, P., Briois, C., Calmonte, U., Combi, M., Keyser, J. D., Eberhardt, P., Fiethe, B., Fuselier, S. A., Galand, M., Gasc, S., Gombosi, T. I., Hansen, K. C., Jc"kel, A., Keller, H. U., Kopp, E., Korth, A., Kührt, E., Roy, L. L., Mall, U., Marty, B., Mousis, O., Neefs, E., Owen, T., Rème, H., Rubin, M., Sémon, T., Tornow, C., Tzou, C.-Y., Waite, J. H., and Wurz, P.: Time variability and heterogeneity in the coma of 67P/ChuryumovGerasimenko, Science, 347, 6220, doi:10.1126/science.aaa0276, 2015.

Ip, W. H. and Axford, W. I.: The formation of a magnetic-field-free cavity at comet Halley, Science, 325, 418-419, 1987.

Israelevich, P. L. and Ershkovich, A. I.: Induced magnetosphere of comet Halley 2. Magnetic field and electric currents, J. Geophys. Res., 99, 21225-21232, 1994.

Israelevich, P. L., Neubauer, F. M., and Ershkovich, A. I.: The induced magnetosphere of comet Halley: interplanetary magentic field during Giotto encounter, J. Geophys. Res., 99, 6575-6583, 1994.

Kirsch, E., McKenna-Lawlor, S., Daly, P., Korth, A., Neubauer, F. M., O'Sullivan, D., Thompson, A., and Wenzel, K. P.: Evidence for the field line reconnection process in the particle and magnetic field measurements obtained during the Giotto-Halley encounter, Ann. Geophys., 7, 107-113, 1989.

Kirsch, E., Daly, P., Ip, W.-H., McKenna-lawlor, S., and Neubauer, F. M.: Particle observations by EPA/EPONA during the outbound pass of Giotto from Comet Halley and their relationship to large scale magnetic field irregularities, Ann. Geophys., 8, 455-462, 1990.

Koenders, C., Glassmeier, K.-H., Richter, I., Motschmann, U., and Rubin, M.: Revisiting cometary bow shock positions, Planet. Space Sci., 87, 85-95, doi:10.1016/j.pss.2013.08.009, 2013.

Koenders, C., Glassmeier, K.-H., Richter, I., Ranocha, H., and Motschmann, U.: Dynamical features and spatial structures of the plasma interaction region of 67P/ChuryumovGerasimenko and the solar wind, Planet. Space Sci., 105, 101116, doi:10.1016/j.pss.2014.11.014, 2014.

Lucek, E. A., Dunlop, M. W., Balogh, A., Cargill, P., Baumjohann, W., Georgescu, E., Haerendel, G., and Fornacon, G.-H.: Identification of magnetosheath mirror modes in Equator-S magnetic field data, Ann. Geophys., 17, 1560-1573, doi:10.1007/s00585999-1560-9, 1999.

McComas, D. J., Gosling, J. T., Russell, C. T., and Slavin, J. A.: Magnetotails at unmagentized bodies: comparison of comet Giacobini-Zinner and Venus, J. Geophys. Res., 92, 1011110117, 1987.

McPherron, R. L., Russell, C. T., and Coleman, P. J.: Fluctuating magnetic fields in the magnetosphere, II., ULF waves, Space Sci. Rev., 13, 411-454, 1972.

Neubauer, F. M., Glassmeier, K. H., Pohl, M., Raeder, J., Acuna, M. H., Burlaga, L. F., Ness, N. F., Musmann, G., Mariani, F., Wallis, M. K., Ungstrup, E., and Schmidt, H. U.: First results from the Giotto magnetometer experiment at comet Halley, Nature, 321, 352-355, 1986.
Neubauer, F. M., Marschall, H., Pohl, M., Glassmeier, K.-H., Musmann, G., Mariani, F., Acuna, H. G., Burlaga, L. F., Ness, N. F., Wallis, M. K., Schmidt, H. U., and Ungstrup, E.: First results from the Giotto magnetometer experiment during the P/GriggSkjellerup encounter, Astron. Astrophys., 268, L5-L8, 1993.

Nilsson, H., Lundin, R., Lundin, K., Barabash, S., Borg, H., Norberg, O., Fedorov, A., Sauvaud, J.-A., Koskinen, H., Kallio, E., Riihelä, P., and Burch, J. L.: RPC-ICA: The Ion Composition Analyzer of the Rosetta Plasma Consortium, Space Sci. Rev., 128, 671-695, doi:10.1007/s11214-006-9031-z, 2006.

Nilsson, H., Stenberg Wieser, G., Behar, E., Wedlund, C. S., Gunell, H., Yamauchi, M., Lundin, R., Barabash, S., Wieser, M., Carr, C., Cupido, E., Burch, J. L., Fedorov, A., J.-A. Sauvaud, Koskinen, H., Kallio, E., J.-P. Lebreton, Eriksson, A., Edberg, N., Goldstein, R., Henri, P., Koenders, C., Mokashi, P., Nemeth, Z., Richter, I., Szego, K., Volwerk, M., Vallat, C., and Rubin, M.: Birth of a comet magnetosphere: A spring of water ions, Science, 347, aaa0571-1-aaa0571-4, doi:10.1126/science.aaa0571, 2015a.

Nilsson, H., Wieser, G. S., Behar, E., Wedlund, C. S., Kallio, E., Gunell, H., Edberg, N. J. T., Eriksson, A. I., Yamauchi, M., Koenders, C., Wieser, M., Lundin, R., Barabash, S., Mandt, K., Burch, J. L., Goldstein, R., Mokashi, P., Carr, C., Cupido, E., Fox, P. T., Szego, K., Nemeth, Z., Fedorov, A., Sauvaud, J.A., Koskinen, H., Richter, I., Lebreton, J.-P., Henri, P., Volwerk, M., Vallat, C., and Geiger, B.: Evolution of the ion environment of comet 67P/Churyumov-Gerasimenko - Observations between 3.6 and 2.0 AU, Astron. Astrophys., 583, aa26142-15, doi:10.1051/0004-6361/201526142, 2015b.

Opitz, A., Karrer, R., Wurz, P., Galvin, A., Bochsler, P., Blush, L., Daoudi, H., Ellis, L., Farrugia, C., Giammanco, C., Kistler, L., Klecker, B., Kucharek, H., Lee, M., Möbius, E., Popecki, M., Sigrist, M., Simunac, K., Singer, K., Thompson, B., and Wimmer-Schweingruber, R.: Temporal Evolution of the Solar Wind Bulk Velocity at Solar Minimum by Correlating the STEREO A and B PLASTIC Measurements, Sol. Phys., 256, 365-377, doi:10.1007/s11207-008-9304-7, 2009.

Opitz, A., Fedorov, A., Wurz, P., Szego, K., Sauvaud, J.-A., Karrer, R., Galvin, A., Barabash, S., and Ipavich, F.: SolarWind Bulk Velocity Throughout the Inner Heliosphere from Multi-Spacecraft Measurements, Sol. Phys., 264, 377-382, doi:10.1007/s11207-010-9583-7, 2010.

Price, C. P., Swift, W., and Lee, L.-C.: Numerical Simulation of Nonoscillatory Mirror Waves at the Earth's Magnetosheath, J. Geophys. Res., 91, 101-112, 1986.

Raeder, J., Neubauer, F. M., Ness, N. F., and Burlaga, L. F.: Macroscopic perturbations of the IMF by P/Halley as seen by the Giotto magnetometer, Astron. Astrophys., 187, 61-64, 1987.

Reinhard, R.: The Giotto encounter with comet Halley, Nature, 321, 313-318, 1986.

Richter, I., Koenders, C., Auster, H.-U., Frühauff, D., Götz, C., Heinisch, P., Perschke, C., Motschmann, U., Stoll, B., Altwegg, K., Burch, J., Carr, C., Cupido, E., Eriksson, A., Henri, P., Goldstein, R., Lebreton, J.-P., Mokashi, P., Nemeth, Z., Nilsson, H., Rubin, M., Szegö, K., Tsurutani, B. T., Vallat, C., Volwerk, M., and Glassmeier, K.-H.: Observation of a new type of low-frequency waves at comet 67P/Churyumov-Gerasimenko, Ann. Geophys., 33, 1031-1036, doi:10.5194/angeo-33-10312015, 2015. 
Riedler, W., Schwingenschuh, K., Yeroshenko, Y. E., Styashkin, V. A., and Russell, C. T.: Magnetic field observations in comet Halley's coma, Nature, 321, 288-289, 1986.

Rotundi, A., Sierks, H., Della Corte, V., Fulle, M., Gutierrez, P., Lara, L., Barbieri, C., Lamy, P., Rodrigo, R., Koschny, D., Rickman, H., Keller, H., López-Moreno, J., Accolla, M., Agarwal, J., A’Hearn, M., Angrilli, N. A. F., Barucci, M., Bertaux, J.-L., Bertini, I., Bodewits, D., Bussoletti, E., Colangeli, L., Cosi, M., Cremonese, G., Crifo, J.-F., Da Deppo, V., Davidsson, B., Debei, S., with F. Esposito, M. D. C., Ferrari, M., Fornasier, S., Giovane, F., Gustafson, B., Green, S., Groussin, O., Grün, E., Güttler, C., Herranz, M., Hviid, S., Ip, W., Ivanovski, S., Jerónimo, J., Jorda, L., Knollenberg, J., Kramm, R., Kührt, E., and. M. Lazzarin, M. K., Leese, M., López-Jiménez, A., Lucarelli, F., Lowry, S., Marzari, F., Epifani, E., McDonnell, J., Mennella, V., Michalik, H., Molina, A., Morales, R., Moreno, F., Mottola, S., Naletto, G., Oklay, N., Ortiz, J., Palomba, E., Palumbo, P., Perrin, J.-M., Rodríguez, J., Sabau, L., Snodgrass, C., Sordini, R., Thomas, N., Tubiana, C., Vincent, J.-B., Weissman, P., Wenzel, K.-P., Zakharov, V., and Zarnecki, J.: Dust measurements in the coma of comet 67P/Churyumov-Gerasimenko inbound to the Sun, Science, 347, aaa3905, doi:10.1126/science.aaa3905, 2015.

Sauer, K., dubinin, E., Baumgärtel, K., and Tarasov, V.: Lowfrequency electromagnetic waves and instabilities within the Martian bi-ion plasma, Earth Planets Space, 50, 269-278, doi:10.1186/BF03352113, 1998.

Schmid, D., Volwerk, M., Plaschke, F., Vörös, Z., Zhang, T. L., Baumjohann, W., and Narita, Y.: Mirror mode structures near Venus and Comet P/Halley, Ann. Geophys., 32, 651-657, doi:10.5194/angeo-32-651-2014, 2014.
Smith, E. J., Tsurutani, B. T., Slavin, J. A., Jones, D. E., Siscoe, G. L., and Mendis, D. A.: ICE Encounter with Giacobini-Zinner: Magnetic Field Observations, Science, 232, 382-385, 1986.

Sonnerup, B. U. Ö. and Scheible, M.: Minimum and maximum variance analysis, in: Analysis Methods for Multi-Spacecraft Data, edited by Paschmann, G. and Daly, P., pp. 185-220, ESA, Noordwijk, the Netherlands, 1998.

Tao, C., Kataoko, R., Fukunishi, H., Takahashi, Y., and Yokokama, T.: Magnetic field variations in the Jovian magnetotail induced by solar wind dynamic pressure enhancements, J. Geophys. Res., 110, A11208, doi:10.1029/2004JA010959, 2005.

Trotignon, J. G., Michau, J. L., Lagoutte, D., Chabassièreand G. Chalumeau, M., Colin, F., Décréau, P. M. E., Geiswiller, J., Gille, P., Grard, R., Hachemi, T., Hamelin, M., Eriksson, A., Laakso, H., Lebreton, J. P., Mazelle, C., Randriamboarison, O., Schmidt, W., Smit, A., Telljohann, U., and Zamora, P.: RPCMIP: the Mutual Impedance Probe of the Rosetta Plasma Consortium, Space Sci. Rev., 128, 713-728, doi:10.1007/s11214-0069005-1, 2006.

Tsurutani, B. T., Smith, E. J., Anderson, R. R., Ogilvie, K. W., Scudder, J. D., Baker, D. N., and Bame, S. J.: Lion roars and nonoscillatory drift mirror waves in the magnetosheath, J. Geophys. Res., 87, 6060-6072, 1982.

Tsurutani, B. T., Lakhina, G. S., Smith, E. J., Buti, B., Moses, S. L., Coroniti, F. V., Brinca, A. L., Slavin, J. A., and Zwickl, R. D.: Mirror mode structures and ELF plasma waves in the GiacobiniZinner magnetosheath, Nonlin. Processes Geophys., 6, 229-234, doi:10.5194/npg-6-229-1999, 1999.

Verigin, M. I., Axford, W. I., Gringauz, K. I., and Richter, A. K.: Acceleration of cometary plasma in the vicinity of comet Halley associated with an interplanetary magnetic field polarity change, Geophys. Res. Lett., 14, 987-990, 1987.

Volwerk, M., Glassmeier, K.-H., Delva, M., Schmid, D., Koenders, C., Richter, I., and Szegö, K.: A comparison between VEGA 1, 2 and Giotto flybys of comet 1P/Halley: implications for Rosetta, Ann. Geophys., 32, 1441-1453, doi:10.5194/angeo-321441-2014, 2014. 